\title{
Reply to: Extracting Kondo temperature of strongly- correlated systems from the inverse local magnetic susceptibility
}

\author{
Xiaoyu Deng (10 ${ }^{1 凶}$, Katharina M. Stadler ${ }^{2}$, Kristjan Haule (i) ${ }^{1}$, Seung-Sup B. Lee ${ }^{2}$, Andreas Weichselbaum ${ }^{2,3}$, \\ Jan von Delft (iD ${ }^{2} \&$ Gabriel Kotliar ${ }^{1,3 凶}$
}

REPLYING to A. A. Katanin Nature Communications https://doi.org/10.1038/s41467-021-21641-2 (2021).

$\mathrm{n}$ his comment $^{1}$, Katanin reanalyzes our LDA + DMFT results $^{2}$ for the temperature-dependent static local spin susceptibility of $\mathrm{Sr}_{2} \mathrm{RuO}_{4}$ and $\mathrm{V}_{2} \mathrm{O}_{3}$ fitting them to a Curie-Weiss (CW) form, $\chi(T) \simeq a /(T+\theta)$. Invoking Wilson's analysis ${ }^{3}$ of the impurity susceptibility of the spin-1/2 one-channel Kondo model $(1 \mathrm{CKM})$ in the wide-band limit, he extracts spin Kondo temperatures using $T_{\mathrm{K}}=\theta / \sqrt{2}$, obtaining $T_{\mathrm{K}}=350 \mathrm{~K}$ and $100 \mathrm{~K}$ for $\mathrm{Sr}_{2} \mathrm{RuO}_{4}$ and $\mathrm{V}_{2} \mathrm{O}_{3}$, respectively. Noting that these are significantly smaller than the scales $T_{\mathrm{sp}}^{\text {onset }}=2300 \mathrm{~K}$ and $1000 \mathrm{~K}$ reported in ref. ${ }^{2}$, he argues that our $T_{\mathrm{sp}}^{\text {onset }}$ scales "do not characterize the screening process".

We welcome Katanin's use of our data. However, his implication that our $T_{\mathrm{sp}}^{\text {onset }}$ was intended to fully characterize the screening process is misleading. Our work uses the full susceptibility vs. temperature curve to describe properly spin screening, not just a single number. Furthermore, our $T_{\mathrm{sp}}^{\text {onset }}$ was defined to characterize the high-temperature onset of spin screening, whereas his $T_{\mathrm{K}}$ characterizes the $\mathrm{CW}$ regime found at intermediate (i.e., lower) temperatures. The fact that $T_{\mathrm{K}}$ is much smaller than $T_{\mathrm{sp}}^{\text {onset }}$ is therefore not surprising but natural.

We agree with Katanin that, for Hund metals in general and $\mathrm{Sr}_{2} \mathrm{RuO}_{4}$ in particular, it is reasonable to approximate $\chi(T)$, using results of a Kondo impurity which features a CW law at intermediate temperatures. (In the Supplementary material we analyze $\chi(T)$ taken data from DMFT studies of the model Hund system used in ref. ${ }^{2}$.) However, this was already well known. For $\mathrm{Sr}_{2} \mathrm{RuO}_{4}$, a comparison to the exact solution of a (fully screened) spin-1 Kondo model impurity model was carried out in the inset of Fig. 3a of ref. ${ }^{4}$ (ref. 17 of ref. $^{2}$ ), reproduced as Fig. 1(left) below, and a CW fit of that data was published in Fig. 2a of ref. ${ }^{5}$ (cited as ref. 5 of ref. ${ }^{2}$ ). We reproduce it as Fig. 1(right) below. Since $\mathrm{Sr}_{2} \mathrm{RuO}_{4}$ and $\mathrm{V}_{2} \mathrm{O}_{3}$ have an atomic ground state configuration spin closer to 1 than $1 / 2$, the use of a (fully screened) spin-1 Kondo model is more reasonable. Furthermore, when interpreting LDA+DMFT results, it is preferable to use definitions of the Kondo scale that rely on the low-temperature portion of the susceptibility curve, as was done in refs. ${ }^{4,10}$, as opposed to the high-temperature portion as in Katanin's proposal to characterize spin screening. We elaborate on these points and propose a simple way to characterize spin crossovers of Hund metals below.

Since Katanin's comment invokes the 1CKM, we start by summarizing some of its well-established properties ${ }^{3,6,7} \cdot \chi(T)$ exhibits a very broad crossover, from Curie-like high-temperature behavior governed by a local-moment fixed point describing a free spin, to Pauli-like low-temperature behavior governed by a Fermi-liquid fixed point describing a fully screened spin. A proper description of this crossover requires a crossover scaling function, $F\left(T / T_{\mathrm{K}}\right)$ and a crossover scale, the Kondo scale $T_{\mathrm{K}}$, with $\chi(T)=F\left(T / T_{\mathrm{K}}\right) / T$. Wilson showed that $F(x)$ is universal under the assumptions of very weak impurity-bath coupling and infinite bandwidth, and computed it numerically. There are multiple ways of defining $T_{\mathrm{K}}$, evoking the behavior of $F(\mathrm{x})$ for either $x \gg 1, x \simeq 1$, or $x \ll 1$, yielding $T_{\mathrm{K}}$ values differing only by factors of order unity. Wilson's definition of $T_{\mathrm{K}}$ (adopted by Katanin), denoted $T_{\mathrm{W}}$ here, evokes the $x \gg 1$ limit. For high temperatures, $T \gtrsim 16 T_{\mathrm{W}}$, he found $\chi(T) \simeq 1 /(4 T)\left[1-1 / \ln \left(T / T_{\mathrm{W}}\right)+O\left(1 / \ln ^{3}\left(T / T_{\mathrm{W}}\right)\right]\right.$, with $T_{\mathrm{W}}$ defined such that the coefficient of $1 / \ln ^{2}\left(T / T_{\mathrm{W}}\right)$ vanishes. For intermediate temperatures, $0.5 T_{\mathrm{W}}<T<16 T_{\mathrm{W}}$, his numerical results are well approximated by a CW form, with $a=0.17$ and $\theta \sim \sqrt{2} T_{\mathrm{W}} 3,6$ (as used by Katanin). At zero temperature, Wilson found $\chi(0) \sim 0.103 / T_{\mathrm{W}}$ (Eq. (IX.91) of ref. ${ }^{3}$ ). Subsequent BetheAnsatz (BA) calculations of the scaling function 6,7 matched Wilson's numerical results. Analogous results have been obtained for fully screened Kondo models with higher spins ${ }^{8,9}$.The BA works showed that the curve $\chi(T)$ vs. $T / T_{\mathrm{K}}$ depends on the spin $S$, with $\chi(T) \propto S(S+1)] /(3 T)$ for $T / T_{\mathrm{K}} \gg 1$ and $\chi(T) \propto S$ for $T / T_{\mathrm{K}} \ll 1$. The Kondo scales defined in these BA works are

\footnotetext{
${ }^{1}$ Department of Physics and Astronomy, Rutgers University, Piscataway, NJ, USA. ${ }^{2}$ Physics Department, Arnold Sommerfeld Center for Theoretical Physics and Center for NanoScience, Ludwig-Maximilians-Universitat München, München, Germany. ${ }^{3}$ Condensed Matter Physics and Materials Science

Department, Brookhaven National Laboratory, Upton, NY, USA.凶email: xiaoyu.deng@gmail.com; kotliar@physics.rutgers.edu
} 

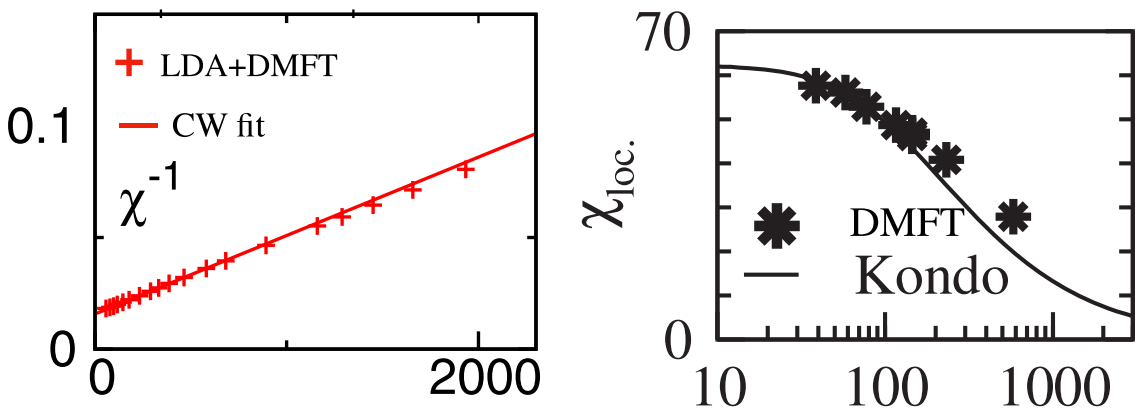

Fig. 1 Earlier work comparing Kondo impurity model with LDA+DMFT results for $\mathbf{S r}_{\mathbf{2}} \mathbf{R u O}_{\mathbf{4}}$. Left: $1 / \chi(T)$ versus $T$, with $L D A+D M F T$ results for $\mathrm{Sr}_{2} \mathrm{RuO}{ }_{4}$ (red symbols) and a Curie-Weiss fit (straight red line) reproduced from the inset of Fig. 2a of ref. ${ }^{5}$. Right: Bethe-Ansatz results for the spin-1,2-channel Kondo model $\chi(T)$ vs. $T$ with $T_{\text {BA }}=240 \mathrm{~K}$ (solid line) in good agreement with the LDA + DMFT results for $\mathrm{Sr}_{2} \mathrm{RuO}_{4}$ (black symbols) reproduced from inset of Fig. 3a of ref. ${ }^{4}$.
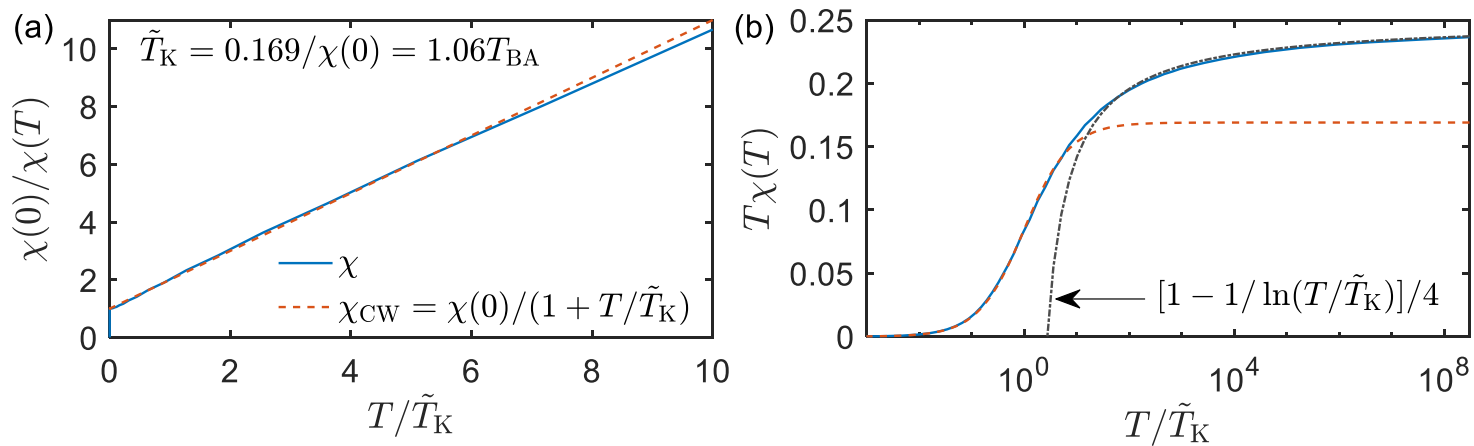

Fig. 2 Two representations of the impurity susceptibility $\chi(T)$ as defined by Wilson ${ }^{3}$ (blue lines), for the 1CKM in the wide-band limit, computed using the numerical renormalization group (NRG). a The Curie-Weiss form (red dashed line) works reasonably well for intermediate temperatures, but (b) not at all for large temperatures, $T / \tilde{T}_{\mathrm{K}} \gg 1$, where logarithmic corrections are large (black dash-dotted line).

independent of spin as in Eq. (21) of ref. ${ }^{9}: T_{\mathrm{BA}}=S /[\pi \chi(0)]$, with $T_{\mathrm{BA}} / T_{\mathrm{W}}=1.55$ for $S=1 / 2$.

In ref. ${ }^{2}$, we used a strategy similar to Wilson's: we identified the regions where the behavior of $\chi_{\text {spin }}(T)$ and $\chi_{\text {orb }}(T)$ is governed by atomic physics or Fermi-liquid theory and numerically computed the crossover function bridging them. We defined two scales for the onset and completion of spin screening, $T_{\mathrm{sp}}^{\text {onset }}$ and $T_{\mathrm{sp}}^{\mathrm{cmp}}$ as the temperatures above or below which $\chi_{\mathrm{spin}}(T)$ shows pure Curie behavior $(\sim 1 / T)$ or pure Pauli behavior $\left(\sim\right.$ const), respectively, and similarly $T_{\text {orb }}^{\text {onset }}$ and $T_{\text {orb }}^{\text {cmp }}$ for orbital screening. Our $T_{\mathrm{sp}}^{\text {onset }}$ and $T_{\mathrm{sp}}^{\mathrm{cmp}}$ scales are similar in spirit to Wilson's $16 T_{\mathrm{W}}$ and $0.5 T_{\mathrm{W}}$. So even within the 1CKM framework, an extraction of $T_{\mathrm{W}}$ from our results, using $T_{\mathrm{W}} \simeq T_{\mathrm{sp}}^{\text {onset }} / 16$, would yield $2300 \mathrm{~K} / 16 \simeq 140 \mathrm{~K}$ for $\mathrm{Sr}_{2} \mathrm{RuO}_{4}$ and $1000 \mathrm{~K} / 16 \simeq 60 \mathrm{~K}$, and the order of magnitude discrepancy claimed by Katanin disappears.

Contrary to this crude estimate, in ref. ${ }^{2}$ we did not assume $T_{\mathrm{sp}}^{\text {onset }}$ to be proportional to a single Kondo scale since even for an impurity model without DMFT self-consistency, $T_{\mathrm{sp}}^{\text {onset }}$ is known to be affected by energy scales not present in the wide-band 1CKM (e.g., a finite bandwidth or a finite charging energy), since such scales cut off high-temperature logarithmic corrections [cf. ref. ${ }^{10}$, Fig. 2b, c]. This is even more important for Mott systems, where the emergence of a quasi-particle resonance with decreasing temperatures affects the bath bandwidth via DMFT self-consistency.

In ref. ${ }^{2}$, we supplemented our LDA+DMFT study of actual materials by DMFT studies of a multi-orbital model Hamiltonian, again computing $\chi(T)$ numerically. We found signatures distinguishing Mottness and Hundness (such as $T_{\text {spin }}^{\text {onset }} \simeq T_{\text {orb }}^{\text {onset }}$ for the former but $T_{\text {spin }}^{\text {onset }}<T_{\text {orb }}^{\text {onset }}$ for the latter) similar to those found in the materials. We defined a Kondo scale $T_{\mathrm{K} \text {.spin }}^{\mathrm{dyn}}\left(\right.$ denoted $T_{\mathrm{K}}$ in ref. ${ }^{2}$ ) through the imaginary part of the $T=0$ dynamical spin susceptibility, $\chi^{\prime \prime}\left(\omega=T_{\mathrm{K}, \text { spin }}^{\mathrm{dyn}}\right)=$ maximal. $T_{\mathrm{K}, \mathrm{spin}}^{\mathrm{dyn}}$ characterizes the intermediate region, with $T_{\text {spin }}^{\mathrm{cmp}}<T_{\mathrm{K}, \text { spin }}^{\mathrm{dyn}}<T_{\text {spin }}^{\text {onset }}$. It is shown as a red line in Fig. $5 \mathrm{~b}$ of ref. ${ }^{2}$, yielding $T_{\mathrm{K}, \text { spin }}^{\mathrm{d}}=0.12 t=600 \mathrm{~K}$ for our Hund system $\mathrm{H} 1$ mimicking $\mathrm{Sr}_{2} \mathrm{RuO}_{4}$, and $T_{\mathrm{K}, \text { spin }}^{\mathrm{dyn}}=0.04 t=200 \mathrm{~K}$ for our Mott system M1 mimicking $\mathrm{V}_{2} \mathrm{O}_{3}$ (using the conversion factor $t=5000 \mathrm{~K}$ stated in Fig. 1).

We take Katanin's comment as an incentive to propose a standardized scheme for extracting a Kondo scale, $\tilde{T}_{\mathrm{K}}$, from a computed $\chi(T)$ curve. Our scheme (i) does not involve a fit to predictions of a specific impurity model, since in general it is unclear which impurity model to compare to, and (ii) uses the $x \leq 1$ part of the crossover scaling function, since it is more universal than the $x \gg 1$ part $^{8-10}$; and (iii) reduces to impurity-model results when these are applicable. We propose to define $T_{\mathrm{K}}$ through the relation $\chi\left(\widetilde{T}_{\mathrm{K}}\right) / \chi(0)=1 / 2$. (If $\chi(0)$ is not known but $\chi(T)$ shows CW-type behavior at intermediate temperatures, $\chi(0)$ can be estimated by linear extrapolation of $1 / \chi(T)$ vs. $T$ to zero temperature.) This definition ensures that $T_{\mathrm{sp}}^{\text {comp }}<\tilde{T}_{\mathrm{K}}<T_{\mathrm{sp}}^{\text {onset }}$, as it should. For the CW form it yields $\tilde{T}_{\mathrm{K}}=\theta$. For the 1CKM, NRG computations (Fig. 2) show that $\tilde{T}_{\mathrm{K}}=0.169 / \chi(0)=1.06 T_{\mathrm{BA}}=1.64 T_{\mathrm{W}}$. For the materials $\mathrm{Sr}_{2} \mathrm{RuO}_{4}$ and $\mathrm{V}_{2} \mathrm{O}_{3}$ studied in ref. ${ }^{2}$, Katanin's CW extraction of $\theta$-values implies $\widetilde{T}_{\mathrm{K}}=574 \mathrm{~K}$ or $164 \mathrm{~K}$, respectively. This illustrates, yet again, the main point of this reply: the Kondo scale is generically much smaller than $T_{\mathrm{sp}}^{\text {onset }}$, and it is misleading to conflate these two scales.

\section{Data availability}

The authors declare that the data supporting the findings of this study are available from the authors. 
Received: 27 February 2020; Accepted: 29 January 2021;

Published online: 04 March 2021

\section{References}

1. Katanin, A. A. Extracting Kondo temperature of strongly-correlated systems from the inverse local magnetic susceptibility. Nat. Commun. https://doi.org/ 10.1038/s41467-021-21641-2 (2021).

2. Deng, X. et al. Signatures of Mottness and Hundness in archetypal correlated metals. Nat. Commun. 10, 2721 (2019).

3. Wilson, K. The renormalization group: critical phenomena and the Kondo problem. Rev. Mod. Phys. 47, 773-840 (1975).

4. Mravlje, J. et al. Coherence-incoherence crossover and the massrenormalization puzzles in $\mathrm{Sr}_{2} \mathrm{RuO}_{4}$. Phys. Rev. Lett. 106, 096401 (2011).

5. Mravlje, J. \& Georges, A. Thermopower and entropy: lessons from $\mathrm{Sr}_{2} \mathrm{RuO}_{4}$. Phys. Rev. Lett. 117, 036401 (2016).

6. Tsvelick, A. \& Wiegmann, P. Exact results in the theory of magnetic alloys. Adv. Phys. 32, 453-713 (1983).

7. Andrei, N., Furuya, K. \& Lowenstein, J. H. Solution of the Kondo problem. Rev. Mod. Phys. 55, 331-402 (1983).

8. Desgranges, H.-U. Thermodynamics of the n-channel Kondo problem (numerical solution). J. Phys. C: Solid State Phys. 18, 5481-5486 (1985).

9. Bortz, M. \& Klümper, A. The anisotropic multichannel spin-S Kondo model: calculation of scales from a novel exact solution. Eur. Phys. J. B 40, 25-42 (2004).

10. Hanl, M. \& Weichselbaum, A. Local susceptibility and Kondo scaling in the presence of finite bandwidth. Phys. Rev. B 89, 075130 (2014).

\section{Acknowledgements}

Work by X.D. and G.K. was supported by NSF DMR 1733071. Work by K.H. was supported by NSF DMR 1405303. S.S.B.L., K.M.S., and J.v.D. acknowledge support from the excellence initiative NIM; A.W. was supported by the U.S. Department of Energy, Office of Basic Energy Sciences, under Contract No. DE-SC0012704. We are grateful to Dr. Jernej Mravlje for sharing his data and helpful discussions.

\section{Author contributions}

All the authors X.D., K.M., K.H., S.S.B.L., A.W., J.v.D., and G.K. discussed the comment and participated in the drafting of the response.

\section{Competing interests}

The authors declare no competing interests.

\section{Additional information}

Supplementary information The online version contains supplementary material available at https://doi.org/10.1038/s41467-021-21643-0.

Correspondence and requests for materials should be addressed to X.D. or G.K.

Peer review information Nature Communications thanks Michele Fabrizio and the other, anonymous, reviewer(s) for their contribution to the peer review of this work

Reprints and permission information is available at http://www.nature.com/reprints

Publisher's note Springer Nature remains neutral with regard to jurisdictional claims in published maps and institutional affiliations.

cc (i) Open Access This article is licensed under a Creative Commons Attribution 4.0 International License, which permits use, sharing, adaptation, distribution and reproduction in any medium or format, as long as you give appropriate credit to the original author(s) and the source, provide a link to the Creative Commons license, and indicate if changes were made. The images or other third party material in this article are included in the article's Creative Commons license, unless indicated otherwise in a credit line to the material. If material is not included in the article's Creative Commons license and your intended use is not permitted by statutory regulation or exceeds the permitted use, you will need to obtain permission directly from the copyright holder. To view a copy of this license, visit http://creativecommons.org/ licenses/by/4.0/.

(c) The Author(s) 2021, corrected publication 2023 